CARPATHIAN J. MATH.

Volume 37 (2021), No. 2,

Pages 345 - 354
Online version at https : //www . carpathian. cunbm. utcluj. ro/

Print Edition: ISSN 1584 - 2851; Online Edition: ISSN 1843 - 4401

DOI: https://doi.org/10.37193/CJM.2021.02.19

Dedicated to Prof. Ioan A. Rus on the occasion of his $85^{\text {th }}$ anniversary

\title{
Conversions between generalized metric spaces and standard metric spaces with applications in fixed point theory
}

\author{
ALEXANDRU-DARIUS FILIP
}

\begin{abstract}
In this paper we discuss similar problems posed by I. A. Rus in Fixed point theory in partial metric spaces (Analele Univ. de Vest Timişoara, Mat.-Inform., 46 (2008), 149-160) and in Kasahara spaces (Sci. Math. Jpn., 72 (2010), No. 1, 101-110). We start our considerations with an overview of generalized metric spaces with $\mathbb{R}_{+}$-valued distance and of generalized contractions on such spaces. After that we give some examples of conversions between generalized metric spaces and standard metric spaces with applications in fixed point theory. Some possible applications to theoretical informatics are also considered.
\end{abstract}

\section{INTRODUCTION}

In [28], I. A. Rus presented three interesting open problems in the context of partial metric spaces. Let $(X, p)$ be a complete partial metric space and $d_{p}^{s}: X \times X \rightarrow \mathbb{R}_{+}$be the metric induced by the partial metric $p, d_{p}^{s}(x, y):=2 p(x, y)-p(x, x)-p(y, y)$, for all $x, y \in X$.

Problem 1. If $f:(X, p) \rightarrow(X, p)$ is a generalized contraction, which conditions satisfies $f$ with respect to the metric $d_{p}^{s}$ ?

Problem 2. State fixed point theorems for these new classes of operators on the metric space $\left(X, d_{p}^{s}\right)$.

Problem 3. Use the results of the above problems to give fixed point theorems in a partial metric space.

Similar problems are also considered in the case of dislocated metric spaces.

Some answers to these problems can be found in [1], chapter 7. See also [2], [34].

In this paper we discuss these problems and give some applications. The terminology and notations given in [28], [29] and [9] are followed.

\section{SOME NOTIONS CONCERNING GENERALIZED METRIC SPACES}

There is an impressive number of generalized metric spaces in the fixed point literature, mostly due to the conditions satisfied by the generalized metric. A solution to unify the terminology in the theory of generalized metric spaces was given in [28]. We recall the main terminology and notations, with some additions.

By a generalized metric on a given nonempty set $X$, we understand a functional $d$ : $X \times X \rightarrow \mathbb{R}_{+}$(also called distance functional) which satisfies some axioms. The following

Received: 28.04.2021. In revised form: 02.06.2021. Accepted: 03.06.2021

2010 Mathematics Subject Classification. 54H25, 54Exx, 47H10, 47H09, 68Q25, 68Q55.

Key words and phrases. distance, non-symmetric distance, nonzero self-distance, generalized metric, dislocated metric, partial metric, distance order, operator on generalized metric space, Kasahara space, operator on Kasahara space, fixed point, saturated fixed point principle. 
axioms appear in the definitions of several types of generalized metrics ( [1], [4], [7], [9], [11], [16], [14], [19], [28], [31]):

(i) $d(x, y)=0$ if and only if $x=y$;

$\left(i_{1}\right) d(x, x)=0$, for all $x \in X$;

$\left(i_{2}\right) d(x, y)=0$ implies $x=y$;

$\left(i_{3}\right) d(x, y)=d(y, x)=0$ if and only if $x=y$;

$\left(i_{4}\right) d(x, y)=d(y, x)=0$ implies $x=y$;

$\left(i_{5}\right) d(x, x)=d(y, y)=d(x, y)$ if and only if $x=y$;

$\left(i_{6}\right) d(x, x) \leq d(x, y)$, for all $x, y \in X$;

$\left(i_{7}\right) d(y, y) \leq d(x, y)$, for all $x, y \in X$;

(ii) $d(x, y)=d(y, x)$, for all $x, y \in X$;

$\left(i i_{1}\right) d(x, y) \leq c d(y, x)$, for all $x, y \in X$, with $c>0$;

(iii) $d(x, y) \leq d(x, z)+d(z, y)$, for all $x, y, z \in X$;

$\left(\right.$ iii $\left._{1}\right) d(x, y) \leq d(x, z)+d(y, z)$, for all $x, y, z \in X$;

(iii $\left.i_{2}\right) d(x, y) \leq \max \{d(x, z), d(z, y)\}$, for all $x, y, z \in X$;

(iii $i_{3}$ ) for all $\varepsilon>0, d(x, z) \leq \varepsilon, d(z, y) \leq \varepsilon$ imply $d(x, y) \leq \varepsilon$;

$\left(\right.$ iii $\left._{4}\right) d(x, y) \leq b[d(x, z)+d(z, y)]$, for all $x, y, z \in X$, with $b>1$;

(iii $\left.i_{5}\right) d(x, y) \leq a \max \{d(x, z), d(z, y)\}$, for all $x, y, z \in X$, with $a>1$;

$\left(\right.$ iii $\left._{6}\right) d(x, y) \leq d(x, z)+d(z, y)-d(z, z)$, for all $x, y, z \in X$.

By definition, $d$ is called:

$\odot$ metric if $d$ satisfies $(i)+(i i)+(i i i)$ or $\left(i_{1}\right)+\left(i_{4}\right)+(i i)+(i i i)$;

$\odot$ pseudometric if $d$ satisfies $\left(i_{1}\right)+(i i)+(i i i)$;

$\odot$ quasimetric (or halfmetric) if $d$ satisfies $\left(i_{1}\right)+\left(i_{4}\right)+(i i i)$;

$\odot$ semimetric if $d$ satisfies $(i)+(i i)$;

$\odot$ symmetric if $d$ satisfies $\left(i_{2}\right)+(i i)$;

$\odot$ b-metric if $d$ satisfies $(i)+(i i)+\left(i i i_{4}\right)$;

$\odot$ ultrametric if $d$ satisfies $(i)+(i i)+\left(i i i_{2}\right)$ or $(i)+(i i)+\left(i i_{3}\right)$;

$\odot$ quasiultrametric if $d$ satisfies $(i)+\left(i i_{1}\right)+\left(i i i_{5}\right)$;

$\odot$ quasi-pseudo-ultrametric if $d$ satisfies $\left(i_{1}\right)+\left(i i i_{2}\right)$;

$\odot$ quasi-pseudometric (or premetric) if $d$ satisfies $\left(i_{1}\right)+($ iii $)$;

$\odot$ pseudo-ultrametric if $d$ satisfies $\left(i_{1}\right)+(i i)+\left(i i i_{2}\right)$;

$\odot$ partial metric if $d$ satisfies $\left(i_{5}\right)+\left(i_{6}\right)+(i i)+\left(i i i_{6}\right)$;

$\odot$ dislocated metric (or $d$-metric) if $d$ satisfies $\left(i_{4}\right)+(i i)+($ iii $)$;

$\odot$ dislocated-quasimetric if $d$ satisfies $\left(i_{4}\right)+($ iii $)$;

$\odot$ dislocated-quasi-ultrametric if $d$ satisfies $\left(i_{4}\right)+\left(i i_{2}\right)$;

$\odot$ dislocated-ultrametric if $d$ satisfies $\left(i_{4}\right)+(i i)+\left(i i i_{2}\right)$.

If $X$ is a nonempty set and $d: X \times X \rightarrow \mathbb{R}_{+}$is a generalized metric on $X$, then the pair $(X, d)$ is called generalized metric space. The generalized metric $d$ induces a convergence structure on $X$, denoted here by $\stackrel{d}{\rightarrow}$. Let $\left\{x_{n}\right\}_{n \in \mathbb{N}}$ be a sequence of elements in $X$. The notions of convergent sequence and Cauchy sequence with respect to the generalized metric $d$ will be recalled in the sequel. Due to the large variety of generalized metrics, we will consider several classes of generalized metrics, taking into account the symmetry axiom (i.e., $(i i))$ and the zero self-distance axiom (i.e., $(i)$, or $\left.\left(i_{1}\right) \&\left(i_{4}\right)\right)$ of $d$.

Case 1. If $d$ satisfies the symmetry axiom and $d$ is a zero self-distance (e.g. standard metric, pseudometric, semimetric, $b$-metric, ultrametric, pseudo-ultrametric) then the notions of convergent sequence and Cauchy sequence are the same as in the standard metric spaces, as follows:

$$
x_{n} \stackrel{d}{\rightarrow} x \in X \Leftrightarrow d\left(x_{n}, x\right) \rightarrow 0 \text { as } n \rightarrow \infty .
$$


$\left\{x_{n}\right\}_{n \in \mathbb{N}} \subset X$ is called Cauchy sequence $\Leftrightarrow d\left(x_{n}, x_{m}\right) \rightarrow 0$ as $n, m \rightarrow \infty$.

Case 2. If $d$ satisfies the symmetry axiom and $d$ is a nonzero self-distance (e.g. dislocated metric, partial metric, symmetric, dislocated ultrametric), there exists different and distinct notions regarding convergent sequences and Cauchy sequences. A notion of convergent sequence is defined as in the Case 1. But there are also notions of convergent sequence which derives from the axioms that are satisfied by the generalized metric $d$. For example, in the case of partial metric spaces $(X, p)$, we have

$$
\left\{x_{n}\right\}_{n \in \mathbb{N}} \subset X, x_{n} \stackrel{p}{\rightarrow} x \in X \Leftrightarrow p(x, x)=\lim _{n \rightarrow \infty} p\left(x, x_{n}\right)=\lim _{n, m \rightarrow \infty} p\left(x_{n}, x_{m}\right) .
$$

$\left\{x_{n}\right\}_{n \in \mathbb{N}} \subset X$ is a Cauchy sequence $\Leftrightarrow$ the sequence $\left\{p\left(x_{n}, x_{m}\right)\right\}_{n, m \in \mathbb{N}}$ converges in $(X, p)$.

Case 3. If $d$ does not satisfy the symmetry axiom and $d$ is a zero self-distance (such as quasimetric, quasiultrametric, quasi-pseudo-ultrametric, quasi-pseudometric) then we have the same notions of convergent sequence and Cauchy sequence as in the quasimetric spaces. So, a sequence $\left\{x_{n}\right\}_{n \in \mathbb{N}} \subset X$ is called:

- forward-Cauchy if $d\left(x_{n}, x_{m}\right) \rightarrow 0$ as $m \geq n \rightarrow \infty$;

- backward-Cauchy if $d\left(x_{n}, x_{m}\right) \rightarrow 0$ as $n \geq m \rightarrow \infty$;

- forward convergent to $x \in X$ if it is forward-Cauchy and $d\left(x_{n}, x\right) \rightarrow 0$ as $n \rightarrow \infty$;

- backward convergent to $x \in X$ if it is backward-Cauchy and $d\left(x, x_{n}\right) \rightarrow 0$ as $n \rightarrow \infty$;

- Cauchy if it is forward-Cauchy and backward-Cauchy;

- convergent to $x \in X$ if it is forward-convergent to $x$ and backward convergent to $x$.

In generalized metric spaces $(X, d)$, there are various order relations induced by $d$ on $X$ :

Example 2.1. Let $(X, q)$ be a quasimetric space. Then

$$
\text { for all } x, y \in X, x \leq_{q} y \Leftrightarrow q(x, y)=0 .
$$

Example 2.2. Let $(X, p)$ be a partial metric space. Then

$$
\text { for all } x, y \in X, x \leq_{p} y \Leftrightarrow p(x, x)=p(x, y) \text {. }
$$

These order relations can be used in the fixed point theory in some generalized metric spaces ([7], [14], [33]).

If $f: X \rightarrow X$ is an operator defined on a generalized metric space $(X, d)$, there are several generalized metric conditions which are usually imposed on $f$ with respect to $d$. Due to these conditions, the operator $f$ is called ([16], [31], [9], [1], [21]):

$\left(o_{1}\right)$ contraction with constant $\alpha$ (or $\alpha$-contraction) if there exists a constant $\alpha \in[0,1)$ such that $d(f(x), f(y)) \leq \alpha d(x, y)$, for all $x, y \in X$.

$\left(o_{2}\right)$ graphic contraction if there exists a constant $\alpha \in[0,1)$ such that $d\left(f(x), f^{2}(x)\right) \leq \alpha d(x, f(x))$, for all $x \in X$.

$\left(o_{3}\right)$ Rakotch operator if there exists a decreasing function $\alpha: \mathbb{R}_{+} \rightarrow \mathbb{R}_{+}$such that $\alpha(t)<$ 1 for all $t>0$ and $d(f(x), f(y)) \leq \alpha(d(x, y)) d(x, y)$, for all $x, y \in X$.

$\left(o_{4}\right)$ Caristi operator if there exists a functional $\varphi: X \rightarrow \mathbb{R}_{+}$such that $d(x, f(x)) \leq \varphi(x)-\varphi(f(x))$, for all $x \in X$.

$\left(o_{5}\right) \varphi$-contraction if there exists a comparison function $\varphi: \mathbb{R}_{+} \rightarrow \mathbb{R}_{+}$(i.e. $\varphi$ is increasing and $\varphi^{n}(t) \rightarrow 0$, as $n \rightarrow \infty$, for all $\left.t \in \mathbb{R}_{+}\right)$such that $d(f(x), f(y)) \leq \varphi(d(x, y))$, for all $x, y \in X$.

$\left(o_{6}\right)$ Kannan operator if there exists a constant $k \in\left[0, \frac{1}{2}\right)$ such that $d(f(x), f(y)) \leq k[d(x, f(x))+d(y, f(y))]$, for all $x, y \in X$.

$\left(o_{7}\right)$ Čirič-Reich-Rus operator if there exist two constants $a, b \in \mathbb{R}_{+}$with $a+2 b<1$ such that $d(f(x), f(y)) \leq a d(x, y)+b[d(x, f(x))+d(y, f(y))]$, for all $x, y \in X$.

$\left(o_{8}\right)$ Chatterjea operator if there exists a constant $c \in\left[0, \frac{1}{2}\right)$ such that $d(f(x), f(y)) \leq c[d(x, f(y))+d(y, f(x))]$, for all $x, y \in X$. 
(og) Zamfirescu operator if there exist three constants $a, b, c \in \mathbb{R}_{+}$with $a<1, b<\frac{1}{2}$ and $c<\frac{1}{2}$ such that at least one of the following conditions is true:

$\left(z_{1}\right) d(f(x), f(y)) \leq a d(x, y)$ for all $x, y \in X$;

$\left(z_{2}\right) d(f(x), f(y)) \leq b[d(x, f(x))+d(y, f(y))]$ for all $x, y \in X$;

$\left(z_{3}\right) d(f(x), f(y)) \leq c[d(x, f(y))+d(y, f(x))]$ for all $x, y \in X$.

$\left(o_{10}\right)$ Hardy-Rogers operator if there exist three constants $a, b, c \in \mathbb{R}_{+}$with $a+2 b+2 c \in$ $(0,1)$ such that

$$
d(f(x), f(y)) \leq a d(x, y)+b[d(x, f(x))+d(y, f(y))]+c[d(x, f(y))+d(y, f(x))],
$$

for all $x, y \in X$.

$\left(o_{11}\right)$ Berinde operator (or almost contraction) if there exist two constants $\delta \in[0,1)$ and $L \geq 0$ such that $d(f(x), f(y)) \leq \delta d(x, y)+L d(y, f(x))$, for all $x, y \in X$.

By endowing a generalized metric space $(X, d)$ with another distance functional $\rho$ : $X \times X \rightarrow \mathbb{R}_{+}$, we obtain, under certain conditions, some other mathematical structures, such as Kasahara spaces, introduced by I. A. Rus in [29].

\section{FROM A DislocAted METRIC SPACE TO A METRIC SPACE}

The notion of dislocated metric was introduced by S.G. Matthews in [18] (see also [14]), along with some other interesting results. Let us consider $(X, \rho)$ be a dislocated metric space and $\left\{x_{n}\right\}_{n \in \mathbb{N}}$ be a sequence in $X$. We recall that

$$
x_{n} \stackrel{\rho}{\rightarrow} x \in X \Leftrightarrow \rho\left(x_{n}, x\right) \rightarrow 0 \text { as } n \rightarrow \infty .
$$

$\left\{x_{n}\right\}_{n \in \mathbb{N}}$ is called Cauchy sequence in $(X, \rho) \Leftrightarrow \rho\left(x_{m}, x_{n}\right) \rightarrow 0$ as $m, n \rightarrow \infty$.

A dislocated metric space $(X, \rho)$ is called complete if and only if every Cauchy sequence with elements in $X$ is convergent in $(X, \rho)$.

Any dislocated metric on a nonempty set $X$ generates a standard metric on $X$. Some important results regarding this aspect are given below ([14], [13]).

Proposition 3.1. Let $(X, \rho)$ be a dislocated metric space. Then $\rho$ induces on $X$ the distance functional:

$$
d_{\rho}: X \times X \rightarrow \mathbb{R}_{+}, d_{\rho}(x, y):= \begin{cases}\rho(x, y), & x \neq y \\ 0, & x=y\end{cases}
$$

The following assertions hold:

(1) $d_{\rho}$ is a metric on $X$;

(2) if $\left\{x_{n}\right\}_{n \in \mathbb{N}} \subset X$ is convergent in $(X, \rho)$ then $\left\{x_{n}\right\}_{n \in \mathbb{N}}$ is convergent in $\left(X, d_{\rho}\right)$;

(3) if $\left\{x_{n}\right\}_{n \in \mathbb{N}} \subset X$ is a Cauchy sequence in $(X, \rho)$ then $\left\{x_{n}\right\}_{n \in \mathbb{N}}$ is Cauchy in $\left(X, d_{\rho}\right)$;

(4) $(X, \rho)$ is complete if and only if $\left(X, d_{\rho}\right)$ is complete;

(5) if $f:(X, \rho) \rightarrow(X, \rho)$ is an $\alpha$-contraction then $f:\left(X, d_{\rho}\right) \rightarrow\left(X, d_{\rho}\right)$ is also an $\alpha$ contraction.

From the fixed point theory point of view it is important to remark that, in general, a metric condition with respect to the distance $\rho$ implies the same metric condition with respect to the metric $d_{\rho}$. For example, $\rho(f(x), f(y)) \leq k[\rho(x, f(x))+\rho(y, f(y))]$, for all $x, y \in$ $X$, implies that $d_{\rho}(f(x), f(y)) \leq k\left[d_{\rho}(x, f(x))+d_{\rho}(y, f(y))\right]$, for all $x, y \in X$. In this way we have at least the set-theoretical conclusions which appear in the fixed point theorems: $F_{f} \neq \emptyset, F_{f}=\left\{x^{*}\right\}, F_{f}=F_{f^{n}}=\left\{x^{*}\right\}$, for all $n \in \mathbb{N}^{*}$.

In a dislocated metric space $(X, \rho)$, we have the order relation induced by $\rho$ on $X$ :

$$
\text { for all } x, y \in X, x \leq_{\rho} y \Leftrightarrow \rho(x, x)=\rho(x, y) \text {. }
$$

On the other hand, we have the order relation induced by the metric $d_{\rho}$ on $X$ :

$$
\text { for all } x, y \in X, x \leq_{d_{\rho}} y \Leftrightarrow d_{\rho}(x, y)=0 \Leftrightarrow x=y \text {. }
$$


So, the order relation, $\leq_{\rho}$, induced by the dislocated metric $\rho$, cannot be wielded by the metric $d_{\rho}$.

In the context of metric spaces, we recall the following result, given by I. A. Rus in [30]:

Theorem 3.1 (Saturated principle of contractions in metric spaces). Let $(X, d)$ be a complete metric space and $f: X \rightarrow X$ be an $\alpha$-contraction. Then we have:

(1) There exists $x^{*} \in X$ such that $F_{f^{n}}=\left\{x^{*}\right\}$, for all $n \in \mathbb{N}$.

(2) For all $x \in X, f^{n}(x) \rightarrow x^{*}$ as $n \rightarrow \infty$.

(3) $d\left(x, x^{*}\right) \leq \psi(d(x, f(x)))$, for all $x \in X$, where $\psi(t)=\frac{t}{1-\alpha}, t \geq 0$.

(4) if $\left\{y_{n}\right\}_{n \in \mathbb{N}} \subset X$ with $d\left(y_{n}, f\left(y_{n}\right)\right) \rightarrow 0$ as $n \rightarrow \infty$, then $y_{n} \rightarrow x^{*}$ as $n \rightarrow \infty$.

(5) if $\left\{y_{n}\right\}_{n \in \mathbb{N}} \subset X$ with $d\left(y_{n+1}, f\left(y_{n}\right)\right) \rightarrow 0$ as $n \rightarrow \infty$, then $y_{n} \rightarrow x^{*}$ as $n \rightarrow \infty$.

(6) If $Y \subset X$ is a closed subset such that $f(Y) \subset Y$, then $x^{*} \in Y$. Moreover, if in addition $Y$ is bounded, then $\bigcap_{n \in \mathbb{N}} f^{n}(Y)=\left\{x^{*}\right\}$.

Taking into account these results, we obtain the main result of this section: the saturated principle of contractions in a metric space generated by a dislocated metric.

Theorem 3.2. Let $(X, \rho)$ be a complete dislocated metric space and $f: X \rightarrow X$ be an $\alpha$ contraction with respect to $\rho$. Then we have:

(1) There exists $x^{*} \in X$ such that $F_{f^{n}}=\left\{x^{*}\right\}$, for all $n \in \mathbb{N}$ and $\rho\left(x^{*}, x^{*}\right)=0$.

(2) For all $x \in X, f^{n}(x) \stackrel{\rho}{\rightarrow} x^{*}$ as $n \rightarrow \infty$.

(3) $\rho\left(x, x^{*}\right) \leq \psi(\rho(x, f(x)))$, for all $x \in X$, where $\psi(t)=\frac{t}{1-\alpha}, t \geq 0$.

(4) if $\left\{y_{n}\right\}_{n \in \mathbb{N}} \subset X$ with $\rho\left(y_{n}, f\left(y_{n}\right)\right) \rightarrow 0$ as $n \rightarrow \infty$, then $y_{n} \rightarrow x^{*}$ as $n \rightarrow \infty$.

(5) if $\left\{y_{n}\right\}_{n \in \mathbb{N}} \subset X$ with $\rho\left(y_{n+1}, f\left(y_{n}\right)\right) \rightarrow 0$ as $n \rightarrow \infty$, then $y_{n} \rightarrow x^{*}$ as $n \rightarrow \infty$.

Proof. Since $\rho$ is a dislocated metric on $X, \rho$ induces the metric $d_{\rho}$ on $X$, defined as in (3.1). We have the following conclusions:

(1). By Proposition 3.1, $\left(X, d_{\rho}\right)$ is a complete metric space and $f:\left(X, d_{\rho}\right) \rightarrow\left(X, d_{\rho}\right)$ is $\alpha$-contraction, so, (1) follows from Theorem 3.1.

(2). By Theorem 3.1, $f^{n}(x) \stackrel{d_{\rho}}{\rightarrow} x^{*}$ as $n \rightarrow \infty$, with $\rho\left(x^{*}, x^{*}\right)=0$. This implies that, $f^{n}(x) \stackrel{\rho}{\rightarrow} x^{*}$ as $n \rightarrow \infty$.

(3). By Theorem 3.1 we have that

$$
d_{\rho}\left(x, x^{*}\right) \leq \frac{1}{1-\alpha} d_{\rho}(x, f(x)), \text { for all } x \in X .
$$

Since $\rho\left(x^{*}, x^{*}\right)=0$, we have $d_{\rho}\left(x, x^{*}\right)=\rho\left(x, x^{*}\right)$. So,

$$
\rho\left(x, x^{*}\right) \leq \frac{1}{1-\alpha} d_{\rho}(x, f(x)) \leq \frac{1}{1-\alpha} \rho(x, f(x)) \text {, for all } x \in X .
$$

(4). Let $\left\{y_{n}\right\}_{n \in \mathbb{N}}$ be a sequence in $X$ with $\rho\left(y_{n}, f\left(y_{n}\right)\right) \rightarrow 0$ as $n \rightarrow \infty$. By Proposition 3.1, item $(2), d_{\rho}\left(y_{n}, f\left(y_{n}\right)\right) \rightarrow 0$ as $n \rightarrow \infty$. By Theorem 3.1, item (3), we have

$$
d_{\rho}\left(y_{n}, x^{*}\right) \leq \frac{1}{1-\alpha} d_{\rho}\left(y_{n}, f\left(y_{n}\right)\right) \rightarrow 0 \text { as } n \rightarrow \infty \text {. }
$$

This implies that $y_{n} \stackrel{d_{\rho}}{\rightarrow} x^{*}$ as $n \rightarrow \infty$ and so, $y_{n} \stackrel{\rho}{\rightarrow} x^{*}$, since $\rho\left(x^{*}, x^{*}\right)=0$.

(5). Let $\left\{y_{n}\right\}_{n \in \mathbb{N}}$ be a sequence in $X$ with $\rho\left(y_{n+1}, f\left(y_{n}\right)\right) \rightarrow 0$ as $n \rightarrow \infty$. Then $d_{\rho}\left(y_{n+1}, f\left(y_{n}\right)\right) \rightarrow 0$ as $n \rightarrow \infty$. It follows that $y_{n} \stackrel{d_{\rho}}{\rightarrow} x^{*}$ as $n \rightarrow \infty$. Since $\rho\left(x^{*}, x^{*}\right)=0$ we get that $y_{n} \stackrel{\rho}{\rightarrow} x^{*}$ as $n \rightarrow \infty$.

In a similar way as above, we can extend to dislocated metric spaces the saturated fixed point results given in [30], [27], [23], [32] and [5].

\section{From a PARTiAl METRIC SPACE TO A METRIC SPACE}

A relevant generic example of dislocated metric space is the partial metric space, notion that was introduced by S. G. Matthews in [19] (see also [2], [7], [14], [13], [17], [22]). So, 
each result in the dislocated metric spaces is a result in the partial metric spaces. For example, if $(X, p)$ is a partial metric space, then the functional $d_{p}: X \times X \rightarrow \mathbb{R}_{+}$defined by

where $x, y \in X$, is a metric on $X$.

$$
d_{p}(x, y):= \begin{cases}p(x, y), & \text { if } x \neq y \\ 0, & \text { if } x=y\end{cases}
$$

A partial metric induces another important metric on $X, d_{p}^{s}: X \times X \rightarrow \mathbb{R}$, defined by $d_{p}^{s}(x, y):=2 p(x, y)-p(x, x)-p(y, y)$, for all $x, y \in X$

In what follows we shall call (0)-convergence the convergence induced on $X$ by $p$ as a dislocated metric, i.e.,

$$
x_{n} \stackrel{(0)}{\rightarrow} x \text { as } n \rightarrow \infty \Leftrightarrow p\left(x_{n}, x\right) \rightarrow 0 \text { as } n \rightarrow \infty .
$$

The specificity of a partial metric space $(X, p)$ is defined by the metric $d_{p}^{s}$. The convergence induced on $X$ by $d_{p}^{s}$ will be denoted here by $\stackrel{p}{\rightarrow}$, i.e.,

$$
x_{n} \stackrel{p}{\rightarrow} x \text { as } n \rightarrow \infty \Leftrightarrow x_{n} \stackrel{d_{p}^{s}}{\rightarrow} x \text { as } n \rightarrow \infty .
$$

If $(X, p)$ is a partial metric space, then we have on $X$ three types of convergence of the sequences:

$$
\stackrel{(0)}{\rightarrow}, \stackrel{d_{p}}{\rightarrow} \text { and } \stackrel{p}{\rightarrow} \text {. }
$$

We remark that the convergence, $\stackrel{(0)}{\rightarrow}$, in general, is not an $F$-convergence and $\stackrel{d_{p}}{\rightarrow}, \stackrel{p}{\rightarrow}$ are $F$-convergences.

Now, let $f: X \rightarrow X$ be an operator defined on a partial metric space $(X, p)$. The following question arises: if $f$ is an $\alpha$-contraction with respect to $p$, does it remain an $\alpha$-contraction with respect to $d_{p}^{s}$ and $d_{p}$ ? Some answers are given in the sequel.

Proposition 4.2. Let $(X, p)$ be a partial metric space. If $f: X \rightarrow X$ is an $\alpha$-contraction then $f:\left(X, d_{p}\right) \rightarrow\left(X, d_{p}\right)$ is also an $\alpha$-contraction.

Proposition 4.3 (I. A. Rus [28]). Let $(X, p)$ be a partial metric space. If $f: X \rightarrow X$ is an $\alpha$-contraction then $d_{p}^{s}(f(x), f(y)) \leq \alpha d_{p}^{s}(x, y)+\alpha p(x, x)-p(f(x), f(x))+\alpha p(y, y)-$ $p(f(y), f(y))$, for all $x, y \in X$.

In order to have a relation for $f$ only with respect to the metric $d_{p}^{s}$, we will use the notion of Schröder's pair defined by I. A. Rus in [28]:

Definition 4.1. Let $X$ be a nonempty set, $f: X \rightarrow X$ be an operator and $\psi: X \rightarrow \mathbb{R}_{+}$ be a functional. The pair $(f, \psi)$ is called Schröder's pair if there exists $\alpha \in(0,1)$ such that $\psi(f(x)) \leq \alpha \psi(x)$, for all $x \in X$.

Lemma 4.1. If $(f, \psi)$ is a Schröder's pair, then $F_{f} \subset Z_{\psi}:=\{x \in X \mid \psi(x)=0\}$.

Lemma 4.2. If $x_{0} \in Z_{\psi}$ then $f^{n}\left(x_{0}\right) \in Z_{\psi}$, for all $n \in \mathbb{N}^{*}$.

Theorem 4.3. Let $(X, d)$ be a complete metric space, $\alpha \in(0,1), \psi: X \rightarrow \mathbb{R}_{+}$and $f: X \rightarrow X$ be an operator. We suppose that:

(i) $(f, \psi)$ is a Schröder's pair;

(ii) if $f^{n}(x) \rightarrow x^{*}$, then $\psi\left(f^{n}(x)\right) \rightarrow \psi\left(x^{*}\right)$;

(iii) $d(f(x), f(y)) \leq \alpha d(x, y)+\alpha \psi(x)-\psi(f(x))+\alpha \psi(y)-\psi(f(y))$, for all $x, y \in X$.

Then:

(1) $F_{f}=\left\{x_{f}^{*}\right\}$;

(2) $f^{n}(x) \rightarrow x_{f}^{*}$ as $n \rightarrow \infty$;

(3) $d\left(f^{n}(x), x_{f}^{*}\right) \leq \frac{\alpha^{n}}{1-\alpha} d(x, f(x))+\frac{n \alpha^{n}+(1-n) \alpha^{n+1}}{(1-\alpha)^{2}} \psi(x)$, for all $x \in X$; 
(4) $d\left(x, x_{f}^{*}\right) \leq \frac{1}{1-\alpha} d(x, f(x))+\frac{\alpha}{(1-\alpha)^{2}} \psi(x)$, for all $x \in X$.

We consider now the case of Kannan operators.

Proposition 4.4. Let $(X, p)$ be a partial metric space. If $f: X \rightarrow X$ is a Kannan operator, then $d_{p}^{s}(f(x), f(y)) \leq k\left[d_{p}^{s}(x, f(x))+d_{p}^{s}(y, f(y))\right]+k p(x, x)+(k-1) p(f(x), f(x))+k p(y, y)+$ $(k-1) p(f(y), f(y))$, for all $x, y \in X$.

Proof. The proof follows from the definition of the metric $d_{p}^{s}$.

Theorem 4.4. Let $(X, d)$ be a complete metric space, $k \in\left(0, \frac{1}{2}\right), \psi: X \rightarrow \mathbb{R}_{+}$be a functional and $f: X \rightarrow X$ be an operator. We suppose that:

(i) $(f, \psi)$ is a Schröder's pair, i.e. $\psi(f(x)) \leq k \psi(x)$, for all $x \in X$;

(ii) if $f^{n}(x) \rightarrow x^{*}$, then $\psi\left(f^{n}(x)\right) \rightarrow \psi\left(x^{*}\right)$;

(iii) $d(f(x), f(y)) \leq k[d(x, f(x))+d(y, f(y))]+k \psi(x)+(k-1) \psi(f(x))+k \psi(y)+(k-$ 1) $\psi(f(y))$, for all $x, y \in X$.

Then:

(1) $F_{f}=\left\{x_{f}^{*}\right\}$;

(2) $f^{n}(x) \rightarrow x_{f}^{*}$ as $n \rightarrow \infty$;

(3) $d\left(f^{n}(x), x_{f}^{*}\right) \leq\left(\frac{k}{1-k}\right)^{n} \frac{1-k}{1-2 k} d(x, f(x))+\left(\frac{k}{1-k}\right)^{n-1} \frac{(1+k)^{2}}{1-2 k} \psi(x)$, for all $x \in X$;

(4) $d\left(x, x_{f}^{*}\right) \leq \frac{1-k}{1-2 k} d(x, f(x))+\frac{(1+k)^{2}}{1-2 k} \psi(x)$, for all $x \in X$.

Proof. $(1)+(2)+(3)$. The assumption $($ iii $)$ and the Lemma 4.1 assures that $\operatorname{Card}\left(F_{f}\right) \leq 1$. From the estimation $d\left(f^{j}(x), f^{j+1}(x)\right) \leq\left(\frac{k}{1-k}\right)^{j} d(x, f(x))+\left(\frac{k}{1-k}\right)^{j} \frac{(1+k)^{2}}{k} \psi(x)$, for all $x \in$ $X$ and $j \in \mathbb{N}$, we obtain

$$
d\left(f^{n}(x), f^{n+m}(x)\right) \leq \sum_{j=n}^{n+m-1} d\left(f^{j}(x), f^{j+1}(x)\right) \leq \zeta_{n} d(x, f(x))+\xi_{n} \psi(x)
$$

where $\zeta_{n}:=\left(\frac{k}{1-k}\right)^{n} \frac{1-k}{1-2 k} \rightarrow 0$ as $n \rightarrow \infty$ and $\xi_{n}:=\left(\frac{k}{1-k}\right)^{n-1} \frac{(1+k)^{2}}{1-2 k} \rightarrow 0$ as $n \rightarrow \infty$. From the completeness of the metric $d$, we get that the sequence $\left(f^{n}(x)\right)_{n \in \mathbb{N}}$ is convergent in $(X, d)$. Let $x^{*}=\lim _{n \rightarrow \infty} f^{n}(x)$. By letting $m \rightarrow \infty$ we get $(3)$. On the other hand, we have $d\left(f\left(x^{*}\right), x^{*}\right) \leq d\left(f\left(x^{*}\right), f^{n}(x)\right)+d\left(f^{n}(x), x^{*}\right) \leq k\left[d\left(x^{*}, f\left(x^{*}\right)\right)+d\left(f^{n-1}(x), f^{n}(x)\right)\right]+$ $d\left(f^{n}(x), x^{*}\right)$ and by letting $n \rightarrow \infty$, we get $(1-k) d\left(x^{*}, f\left(x^{*}\right)\right) \leq 0$. Hence $d\left(x^{*}, f\left(x^{*}\right)\right)=0$, and so, $F_{f}=\left\{x^{*}\right\}$.

(4). $d\left(x, x^{*}\right) \leq d(x, f(x))+d\left(f(x), x^{*}\right) \leq \frac{1-k}{1-2 k} d(x, f(x))+\frac{(1+k)^{2}}{1-2 k} \psi(x)$ for all $x \in X$.

Now, let us consider the case of a Caristi operator on a partial metric space $(X, p)$ and the corresponding operator on $\left(X, d_{p}^{s}\right)$.

Let $f:(X, p) \rightarrow(X, p)$ be a $\varphi$-Caristi operator, i.e., $\varphi: X \rightarrow \mathbb{R}_{+}$is an arbitrary functional and $p(x, f(x)) \leq \varphi(x)-\varphi(f(x))$, for all $x \in X$.

If $\varphi$ is lower semi-continuous, then the $\varphi$-Caristi operator $f$ is called a $\varphi$-Caristi-Kirk operator and if $f$ is a $\varphi$-Caristi operator with $f$ continuous with respect to the metric $d_{p}^{s}$, then $f$ is called a $\varphi$-Caristi-Browder operator (see [6]).

In this case we have:

Theorem 4.5. Let $(X, p)$ be a complete partial metric space and $f: X \rightarrow X$ be a $\varphi$-CaristiBrowder operator. If $2 \varphi(x) \geq p(x, x)$, for all $x \in X$, then $F_{f} \neq \emptyset$ and $f:\left(X, d_{p}^{s}\right) \rightarrow\left(X, d_{p}^{s}\right)$ is a weakly Picard operator.

Proof. We have $d_{p}^{s}(x, f(x))=2 p(x, f(x))-p(x, x)-p(f(x), f(x)) \leq 2 \varphi(x)-p(x, x)-$ $[2 \varphi(f(x))+p(f(x), f(x))-2 p(f(x), f(x))] \leq 2 \varphi(x)-p(x, x)-[2 \varphi(f(x))-p(f(x), f(x))]$, 
for all $x \in X$. This implies that $f:\left(X, d_{p}^{s}\right) \rightarrow\left(X, d_{p}^{s}\right)$ is a $\psi$-Caristi-Browder operator, with $\psi: X \rightarrow \mathbb{R}_{+}, \psi(x)=2 \varphi(x)-p(x, x)$. From the Caristi-Browder theorem (see [6]) in $\left(X, d_{p}^{s}\right)$, we have that $F_{f} \neq \emptyset$ and $f$ is a weakly Picard operator with respect to $\stackrel{d_{p}^{s}}{\rightarrow}$.

\section{FROM A DiSLOCATED METRIC SPACE TO A KASAHARA SPACE}

The notions of Kasahara space, generalized Kasahara space and large Kasahara space were introduced by I. A. Rus in [29]. More considerations on these spaces can be found in [8]-[10]. In this paper, by a Kasahara space we understand a mixed structure, denoted by $(X, \stackrel{F}{\rightarrow}, \rho)$, in which the following conditions hold:

(j) $(X, \stackrel{F}{\rightarrow})$ is a Fréchet $L$-space;

(jj) $\rho: X \times X \rightarrow \mathbb{R}_{+}$is a dislocated metric;

$(j j j)$ if $\left\{y_{n}\right\}_{n \in \mathbb{N}}$ is a Cauchy sequence in $(X, \rho)$ then $\left\{y_{n}\right\}_{n \in \mathbb{N}}$ is convergent in $(X, \stackrel{F}{\rightarrow})$.

In what follows, we present a result for the following problem, given by I. A. Rus in [29]: To construct a common fixed point theory on a Kasahara space.

Theorem 5.6. Let $(X, \stackrel{F}{\rightarrow}, \rho)$ be a Kasahara space and $f, g: X \rightarrow X$ be two operators.

We suppose that:

(i) $f, g:(X, \stackrel{F}{\rightarrow}) \rightarrow(X, \stackrel{F}{\rightarrow})$ are continuous;

(ii) there exists $l \in\left(0, \frac{1}{2}\right)$ such that $\rho(f(x), g(y)) \leq l[\rho(x, f(x))+\rho(y, g(y))]$, for all $x, y \in X$.

In these conditions we have:

(1) $F_{f} \cap F_{g}=\left\{x^{*}\right\}$ and $\rho\left(x^{*}, x^{*}\right)=0$;

(2) for each $x_{0} \in X$, the sequence $\left\{x_{n}\right\}_{n \in \mathbb{N}}$ defined by $x_{2 n}=(g \circ f)^{n}\left(x_{0}\right), x_{2 n+1}=f\left(x_{2 n}\right)$, $n \in \mathbb{N}$, converges in $(X, \stackrel{F}{\rightarrow})$ to $x^{*}$;

(3) for each $y_{0} \in X$, the sequence $\left\{y_{n}\right\}_{n \in \mathbb{N}}$ defined by $y_{2 n}=(f \circ g)^{n}\left(y_{0}\right), y_{2 n+1}=g\left(y_{2 n}\right)$, $n \in \mathbb{N}$, converges in $(X, \stackrel{F}{\rightarrow})$ to $x^{*}$;

(4) the operators $f$ and $g$ are graphic contractions;

(5) $\rho\left(f(x), x^{*}\right) \leq \frac{l}{1-l} \rho\left(x, x^{*}\right)$, for all $x \in X$ and $\rho\left(g(x), x^{*}\right) \leq \frac{l}{1-l} \rho\left(x, x^{*}\right)$, for all $x \in X$.

Proof. (1) $+(2)+(3)$. First we remark that if $x_{0} \in F_{f} \cap F_{g}$ then by the assumption (ii) we get $\rho\left(x_{0}, x_{0}\right)=0$. Also, if $x_{0}, x_{1} \in F_{f} \cap F_{g}$ we have that $\rho\left(x_{0}, x_{1}\right) \leq l\left[\rho\left(x_{0}, x_{0}\right)+\rho\left(x_{1}, x_{1}\right)\right]=0$. Since $\rho$ is a dislocated metric, we have that $x_{0}=x_{1}$. So, $\operatorname{Card}\left(F_{f} \cap F_{g}\right) \leq 1$.

For $x_{0} \in X$, let $\left\{x_{n}\right\}_{n \in \mathbb{N}}$ be the sequence in (2). From $(i i)$ we get $\rho\left(x_{1}, x_{2}\right) \leq \frac{l}{1-l} \rho\left(x_{0}, x_{1}\right)$ and by induction, $\rho\left(x_{n}, x_{n+1}\right) \leq\left(\frac{l}{1-l}\right)^{n} \rho\left(x_{0}, x_{1}\right)$, for all $n \in \mathbb{N}^{*}$. From this, it follows that $\left\{x_{n}\right\}_{n \in \mathbb{N}}$ is a Cauchy sequence. Since $(X, \stackrel{F}{\rightarrow}, \rho)$ is a Kasahara space, $\left\{x_{n}\right\}_{n \in \mathbb{N}}$ is a convergent sequence in $(X, \stackrel{F}{\rightarrow})$. Let $x_{n} \stackrel{F}{\rightarrow} x^{*}$ as $n \rightarrow \infty$. From the continuity of $f$ in $(X, \stackrel{F}{\rightarrow})$ and the relation $x_{2 n+1}=f\left(x_{2 n}\right)$, we have that $x^{*} \in F_{f}$. Also, from the continuity of $g$ in $(X, \stackrel{F}{\rightarrow})$ and the relation $x_{2 n+2}=g\left(x_{2 n+1}\right)$, we have that $x^{*} \in F_{g}$.

So, we have (1) and (2). In a similar way, we prove (3).

(4). Let us prove, for example, that $f$ is a graphic contraction. We have that $\rho\left(f^{2}(x), f(x)\right) \leq \rho\left(f^{2}(x), g\left(x^{*}\right)\right)+\rho\left(g\left(x^{*}\right), f(x)\right) \leq l\left[\rho\left(f^{2}(x), f(x)\right)+\rho(x, f(x))\right]$, for all $x \in X$. This implies that $\rho\left(f^{2}(x), f(x)\right) \leq \frac{l}{1-l} \rho(x, f(x))$, for all $x \in X$, i.e., $f$ is a graphic contraction.

(5). We have $\rho\left(f(x), x^{*}\right)=\rho\left(f(x), g\left(x^{*}\right)\right) \leq l \rho(x, f(x)) \leq l\left[\rho\left(x, x^{*}\right)+\rho\left(x^{*}, f(x)\right)\right]$, for all $x \in X$. Hence, $\rho\left(f(x), x^{*}\right) \leq \frac{l}{1-l} \rho\left(x, x^{*}\right)$, for all $x \in X$. In a similar way, we get $\rho\left(g(x), x^{*}\right) \leq \frac{l}{1-l} \rho\left(x, x^{*}\right)$, for all $x \in X$. 
There exists in the literature, large classes of metric conditions imposed on a pair of operators $f, g: X \rightarrow X$ (see [26] and [24]). The problem is to find those for which we can obtain a similar result as Theorem 5.6.

\section{APPLICATIONS TO THEORETICAL INFORMATICS}

Generalized metric spaces are used in the domain of Theoretical Informatics. In many cases, the solution of a problem is being approximated in a recursive process, made by a computational program, designed to solve the problem. This process consist of several steps, each step giving a better approximation of solution than the previous one. The final step of the recursive process, also called the limit-step gives the best approximation of solution.

The notion of order in generalized metric spaces is also applied. It is seen as the amount of information brought by each step of computation in a recursive process. Each step brings a larger amount of information regarding the solution approximation than the precedent step.

Fixed point theorems in generalized metric spaces are used in the study of algorithmic asymptotic complexity, especially in the case when there are more algorithms that can solve a specific problem. It is of great interest to determine the one which uses the least quantity of resources, especially the running time, in order to find the problem solution.

Several applications are present also in the theory of logic programming and artificial intelligence. One of the most difficult problem in these fields is to find a precise meaning of a program, in order to obtain its specification in an independent way, without taking into account the context or the procedural considerations.

More aspects regarding the applications of fixed point results and generalized metric spaces in the theoretical informatics domain can be found in [14], [3], [7], [12], [17], [25], [33], [15], [20].

Acknowledgments. The author is thankful to anonymous reviewers for remarks and suggestions that improved the quality of the paper.

\section{REFERENCES}

[1] Agarwal, P., Jleli, M. and Samet, B., Fixed Point Theory in Metric Spaces, Springer, 2018

[2] Alghamdi, M. A., Shahzad, N. and Valero, O., On fixed point theory in partial metric spaces, Fixed Point Theory Appl., 175 (2012), 1-30

[3] Alghamdi, M. A., Shahzad, N. and Valero, O., Fixed point theorems in generalized metric spaces with applications to computer science, Fixed Point Theory Appl., 2013 (2013:118), 20 pp.

[4] Berinde, V. and Choban, M., Generalized distances and their associated metrics. Impact on fixed point theory, Creat. Math. Inform., 22 (2013), No. 1, 23-32

[5] Berinde, V., Măruşter, Şt. and Rus I. A., Saturated contraction principle for nonself operators, generalizations and applications, Filomat, 31 (2017), No. 11, 3391-3406

[6] Berinde, V. and Rus, I. A., Caristi-Browder operator theory in distance spaces, 1-28. In: Fixed Point Theory and Graph Theory (Alfuraidan, M. R. and Ansari, Q. H., Eds.), Elsevier, 2016

[7] Bukatin, M., Kopperman, R., Matthews, S. and Pajoohesh, H., Partial metric spaces, The American Mathematical Monthly, 116 (2009), 708-718

[8] Filip, A.-D., Fixed point theory in large Kasahara spaces, An. Univ. Vest Timişoara, Ser. Mat.-Inform., 49 (2011), No. 2, 21-36

[9] Filip, A.-D., Fixed Point Theory in Kasahara Spaces, Casa Cărţii de Ştiinţă, Cluj-Napoca, 2015

[10] Filip, A.-D. and Rus, I. A., Fixed point theory for nonself generalized contractions in Kasahara spaces, An. Univ. Vest Timişoara, Ser. Mat.-Inform., 57 (2019), No. 1, 66-76

[11] Fréchet, M., Les espaces abstraits, Gauthier-Villars, Paris, 1928

[12] Frenţiu, M. and Rus, I. A., Metodologia cercetării ştiinţifice în informatică, Presa Univ. Clujeană, Cluj-Napoca, 2014

[13] Haghi, R. H., Rezapour, Sh. and Shahzad, N., Be careful on partial metric fixed point results, Topology Appl., 160 (2013), 450-454 
[14] Hitzler, P., Generalized Metrics and Topology in Logic Programming Semantics, Ph.D. Dissertation, National Univ. of Ireland, 2001.

[15] Khamsi, M. A. and Misane, D., Fixed point theorems in logic programming, Ann. Math. Artificial Intelligence, 21 (1997), 231-243

[16] Kirk, W. A. and Sims, B., (Eds.), Handbook of Metric Fixed Point Theory, Kluwer, 2001

[17] Künzi, H.-P.A., Pajoohesh, H. and Schellekens, M. P., Partial quasi-metrics, Theoretical Computer Sci., 365 (2006), 237-246

[18] Matthews, S. G., Metric domains for completeness, Technical Report 76, Department of Computer Science, University of Warwick, UK, April 1986

[19] Matthews, S. G., Partial metric topology, Ann. New York Acad. Sci., 728 (1994), 183-197.

[20] Matthews, S. and Bukatin, M., An intelligent theory of cost for partial metric spaces, AGI 2012: Artificial General Intelligence, 168-176

[21] Măruşter, Şt. and Rus, I. A., Kannan contractions and strongly demicontractive mappings, Creative Math. Inform., 24 (2015), No. 2, 171-180

[22] Pasicki, L. Partial metric, fixed points, variational principles, Fixed Point Theory, 17 (2016), No. 2, 435-450

[23] Păcurar, M. and Rus, I. A., Fixed point theory for cyclic $\varphi$-contractions, Nonlinear Anal., 72 (2010), No. 3-4, 1181-1187

[24] Petruşel, A. and Rus, I. A., The relevance of a metric condition on a pair of operators in common fixed point theory, In: Metric Fixed Point Theory and Applications (Cho, Y. J. et al., Eds.), Springer, 2021

[25] Romaguera, S. and Valero, O., On the structure of the space of complexity partial functions, International J. Comput. Math., 85 (2008), No. 3-4, 631-640

[26] Rus, I. A., Metrical Fixed Point Theorems, Univ. Cluj-Napoca, Cluj-Napoca, 1979

[27] Rus, I. A., Cyclic representations and fixed points, Ann. T. Popoviciu Seminar Funct. Eq. Approx. Conv., 3 (2005), 171-178

[28] Rus, I. A., Fixed point theory in partial metric spaces, Analele Univ. de Vest Timişoara, Mat.-Inform., 46 (2008), 149-160

[29] Rus, I. A., Kasahara spaces, Sci. Math. Jpn., 72 (2010), No. 1, 101-110

[30] Rus, I. A., Some variants of contraction principle, generalizations and applications, Stud. Univ. Babes-Bolyai, Math., 61 (2016), No.3, 343-358

[31] Rus, I. A., Petruşel, A. and Petruşel, G., Fixed Point Theory, Cluj Univ. Press, Cluj-Napoca, 2008

[32] Rus, I. A. and Şerban, M. A., Basic problems of the metric fixed point theory and the relevance of a metric fixed point theorem, Carpathian J. Math., 29 (2013), No. 2, 239-258

[33] Rutten, J. J. M. M., Elements of generalized ultrametric domain theory, Theoretical Computer Sci., 170 (1996), 349-381

[34] Samet, B., Vetro, C. and Vetro, F., From metric spaces to partial metric spaces, Fixed Point Theory Appl., 5 (2013), 1-11

\author{
BABEŞ-BOLYAI UNIVERSITY, FACULTY OF ECONOMICS AND BUSINESS ADMINISTRATION \\ DEPARTMENT OF STATISTICS-FORECASTS-MATHEMATICS \\ TeOdor Mihali Street, No. 58-60, 400591, Cluj-NapocA, Romania \\ E-mail address: darius.filip@econ.ubbcluj.ro
}

